\title{
Testing for Heterogeneous Treatment Effects in Experimental Data: False Discovery Risks and Correction Procedures
}

\author{
Günther Fink Margaret McConnell Sebastian Vollmer
}

April 2012

\section{Author Contact Information}

Günther Fink

Harvard School of Public Health

665 Huntington Avenue, Boston, MA 02115

Email:gfink@hsph.harvard.edu

Margaret McConnell

Harvard School of Public Health

665 Huntington Avenue, Boston, MA 02115

Email: mmconne@hsph.harvard.edu

Sebastian Vollmer

Harvard School of Public Health \& University of Hannover

9 Bow Street, Cambridge, MA 02138

Email: svollmer@hsph.harvard.edu 


\title{
Testing for Heterogeneous Treatment Effects in Experimental Data: False Discovery Risks and Correction Procedures
}

\begin{abstract}
We review the statistical models applied to test for heterogeneous treatment effects in the recent empirical literature, with a particular focus on data from randomized field experiments. We show that testing for heterogeneous treatment effects is highly common, and likely to result in a large number of false discoveries when conventional standard errors are applied. We demonstrate that applying correction procedures developed in the statistics literature can fully address this issue, and discuss the implications of multiple testing adjustments for power calculations and experimental design.
\end{abstract}




\section{INTRODUCTION}

Multiple testing refers to any instance that involves the simultaneous testing of more than one hypothesis (Joseph P. Romano et al., 2010b). Even though economists have long been aware of the basic problem of conducting inference in the presence of multiple hypotheses, relatively little attention has been given to this issue in the empirical literature. In this paper we investigate one particularly common case of multiple testing in applied econometrics, the search for heterogeneous treatment effects within experimental data.

Few things have shaped empirical work on economics in the last decade as much as the arrival and establishment of randomized controlled trials (RCTs). The number of randomized experiments has grown rapidly across all continents over the past years. The projects listed on the Innovations for Poverty Actions (IPA) and the Abdul Latif Jameel Poverty Action Lab (JPAL) websites suggests that currently more than 100 RCT studies are either planned or in the field (JPAL, 2011); John List's "Field Experiments" website currently lists more than 250 field experiments (John A. List, 2011). One of the main advantages of experiments is the relative simplicity of the statistical analysis required to conduct causal inference. With properly done randomization, estimating causal effects corresponds to a simple conditional or unconditional mean comparison between treatment and control groups, with limited need or scope for more sophisticated empirical models.

Most experiments are designed to estimate the average effect of a given treatment of interest. However, researchers may become interested in the interactions of a treatment with some baseline characteristics of interest during or after data collection, and wish to test for heterogeneous treatment effects ex-post. In some cases, researchers may learn during fieldwork that the magnitude of the treatment effect hinges on a variable measured at baseline. In other cases, researchers may find that the theoretical framework applied has clear predictions regarding the expected behavioral changes across different subgroups of interest.

In order to provide some sense of how frequently papers test for heterogeneous treatment effects in the experimental, we review all articles using field experiment data published in the top 10 
journals according to the 2009 Engemann and Wall ranking (2009) as well as the Journal of Development Economics (the top field journal) from 2005 and 2010. Out of 34 articles we classified as field-experiment-based, 26 (76\%) estimate separate treatment effects for subgroups, and 10 articles (29\%) estimate treatment effects for ten or more subgroups.

While testing for heterogeneous treatment effects through interaction terms or subgroup analyses is clearly desirable, applying traditional standard errors and p-values is not appropriate. Given that each interaction term represents a separate hypothesis beyond the original experimental design, trying out multiple interaction terms corresponds to multiple hypothesis testing, and results in a substantially increased false discovery risk in the empirical analysis.

To illustrate the severity of this issue, we use data from the Programa de Educación, Salud y Alimentación (PROGRESA) in Mexico, and run Monte-Carlo simulations to estimate a large number of heterogeneous treatment effects within the experimental data. We show that any researcher randomly choosing 10 baseline variables as proxy for an underlying characteristic of interest has a $62 \%$ chance of finding at least one variable significant at the $5 \%$ level. Given that the joint (Bernoulli) distribution for 10 independent binary variables implies a cumulative probabilities of finding at least one irrelevant factor significant with $\alpha=0.05$ is $40 \%$, this implies that a majority of significant interactions uncovered in our PROGRESA regressions likely represent false discoveries. To provide a better sense of the study design implications of multiple testing, we compute ex-ante adjustments needed to sample size if researchers plan to investigate one or multiple interaction effects ex-post. We show that the required sample size adjustments are relatively small as long as the number of tested heterogeneous treatment effects is reasonably small.

The multiple hypothesis testing highlighted in this paper issue is not new, and has been faced by researchers in several other disciplines such as genomics or brain imaging. Possibly motivated by applications in these quickly evolving fields, recent statistical research has produced a number of powerful methods to correct for multiple hypothesis testing as summarized in Farcomeni (2008). While most conclusions of the paper apply to almost all empirical work, we have chosen this particular focus for two main reasons. First, heterogeneous treatment effects, even though they 
are very common in empirical work, have not received much attention in the literature on multiple testing and are not even mentioned in the most recent review by Romano, Shaikh and Wolf (Joseph P. Romano et al., 2010a). Second, the design of field experiments allows researchers to consider heterogeneous treatment effects in the power calculations and thus to fully solve the problem of multiple testing prior to the collection of data.

The paper is related to the broader literature on heterogeneous treatment effects discussed in Angrist (2004), Green and Kern (2010) as well as Imai and Strauss (2011). While these papers primarily focus on optimal model specification in the presence of heterogeneous treatment effects, we mostly focus on the multiple-testing issue associated with sequential subgroup testing in this paper.

The rest of the paper is organized as follows: We start with a review of the methods used in recently published papers in the field in section 2 . We discuss the theoretical and empirical distribution of heterogeneous treatment effects using PROGRESA data in Section 3. We introduce the corrections for multiple testing proposed by the statistics literature in Section 4, and analyze the practical implications of the various correction models in Section 5. In Section 6, we discuss the implications of multiple testing for study design and power calculations; section 7 concludes.

\section{LITERATURE REVIEW}

Based on the Engemann and Wall (2009) ranking of Journals, we surveyed articles in the top 10 ranked journals as well as the Journal of Development Economics as the most commonly cited field journal. We use the classification proposed by Harrison and List (2004) as a guide to determine how to classify field experiments. We focus exclusively on "natural field experiments," which Harrison and List describe as experiments where a "non-standard 1 subject pool" makes decisions where there is a "field context in either the commodity, task, or information set that the subjects can use" and "the environment is one where the subjects

\footnotetext{
${ }^{1}$ In the context of experiments in Harrison and List (2004), a standard subject pool would be undergraduates recruited to perform an experiment in a laboratory setting
} 
naturally undertake these tasks and where the subjects do not know that they are in an experiment.” (Glenn W. Harrison and John A. List, 2004, p. 1014)

In order to be considered for our literature review, a paper must present evidence from a study where a treatment intervention is randomly assigned by design of the study. Therefore, natural experiments and field experiments where treatments are not randomly assigned are excluded from our review. ${ }^{2}$ Furthermore, we exclude papers that focus on econometric methods using data from natural field experiments. We refer to the papers that satisfy this definition as "strict" natural field experiments. While this classification may appear restrictive, it yields a clear decision rule allowing for a consistent review of the existing literature of interest. The main point made in this paper clearly applies to a larger set of empirical papers.

Table 1 shows the journal list, as well as the total number of articles, the number of "strict" natural field experiments.

TABLE 1

“Strict” NATURAl Field EXPERIMENTS PUblished 2005-2009

\begin{tabular}{llcc}
\hline $\begin{array}{l}\text { Journal } \\
\text { Rank }\end{array}$ & Journal Name & $\begin{array}{l}\text { Total } \\
\text { Articles }\end{array}$ & $\begin{array}{l}\text { Field } \\
\text { Experiments" }\end{array}$ \\
\hline 1 & & 283 & 11 \\
2 & Quarterly Journal of Economics & 296 & 1 \\
3 & Journal of Political Economy & 420 & 5 \\
4 & Econometrica & 644 & 8 \\
5 & American Economic Review & 292 & 0 \\
6 & Review of Economic Studies & 201 & 0 \\
7 & Journal of Labor Economics & 87 & 0 \\
8 & Journal of Economic Growth & 456 & 1 \\
9 & Review of Economics and Statistics & 498 & 1 \\
10 & Economic Journal & 592 & 5 \\
30 & American Economic Review Papers and Proceedings & 461 & 2 \\
& Journal of Development Economics & 4230 & 34 \\
\hline
\end{tabular}

\footnotetext{
${ }^{2}$ Examples include the analysis of data where subjects were quasi-randomly matched as in studies of choices in speed dating (Fisman et al, 2006), the impact of random roommate assignment and random assignment by lottery (Angrist, Bettenger and Kremer 2006).
} 
Of 4,230 articles surveyed in these 11 journals over the period 2005-2009, 34 articles feature evidence from strict natural field experiments. Some of the reviewed journals did not publish any study based on a field experiment (Journal of Labor Economics and Journal of Economic Growth), while the Quarterly Journal of Economics published more than 10 studies based on experiments fitting our "strict" natural field experiment description over the same period.

In Table 2 we take a closer look at the econometric strategy employed by articles featuring evidence from strict natural field experiments. We do not show the full title of each paper, but rather show the paper's index number, and provide the full listing as well as references in the Appendix. We report two measures of heterogeneous treatment effect testing: the number of subgroups for which treatment effects are estimated, and the number of interaction effects between the treatment variable and baseline characteristics tested.

In order to classify a reported result as a "heterogeneous treatment effect test" we followed a series of rules avoiding double-counting of treatment effects as well as incorrect classifications of regressions reflecting a particular study design. First, we only consider tests reported in the main tables of a paper, and exclude all results either shown in an appendix or only mentioned in the text. Second, we do not count reported tests reflecting the original research design. In many instances, researchers test for increasing effects over time, and also for heterogeneous treatment effects across different geographic sites. While one could argue that different time periods and sites reflect distinct sub-groups, we consider them as separate experiments, and thus do not count them as instances of heterogeneous treatment tests. ${ }^{3}$ We also do not count heterogeneous treatment effect tests based on baseline characteristics that are measured after the experimental randomization, which are very uncommon but raise a different set of statistical concerns.

In some cases, papers combine subgroup analysis with estimates of interactions with treatments. We count the number of subgroups and interactions separately, and simply report the

\footnotetext{
${ }^{3}$ While experiments may vary by time and location, most researchers do not consider differences across sites and over time to be a primary theoretical hypothesis generated by an economic experiment.
} 
total number of subgroups and the total number of interactions. Furthermore, we only count each interaction or subgroup analysis once, even if they are considered for more than one dependent variable. Multiple dependent variable testing is very common in the literature as well, and the associated statistical problem similar to the heterogeneous treatment effects analyzed in this paper (Duflo, Glennester, et al 2008). The complications from considering dependent variables measured across time are discussed in recent work by McKenzie (2010).

Out of the 34 papers analyzed, 21 articles (62\%) estimate separate treatment effects for subgroups, while 16 articles (47\%) estimate interaction effects between the treatment and baseline characteristics. Only 8 articles (24\%) neither estimate interaction effects nor consider the effect of treatment on subgroups. In some cases, testing for heterogeneous treatment effects is extensive: As Table 2 shows, 10 articles (29\%) estimated 10 or more subgroup or interaction effects. Some examples of common interactions or subgroup analyses are sex, age, wealth and education. None of the article corrects (or mentions) multiple hypothesis testing in the empirical analysis. 
TABLE 2

StruCTURE OF EMPIRICAL MODEL IN FIELD EXPERIMENTS

\begin{tabular}{|c|c|c|c|}
\hline $\begin{array}{l}\text { Article } \\
\text { Index }\end{array}$ & $\begin{array}{l}\text { Type(s) of Dependent } \\
\text { Variable(s): Binary, } \\
\text { Continuous or boTh }\end{array}$ & Number of Subsamples & $\begin{array}{l}\text { Number of Interactions } \\
\text { Estimated }\end{array}$ \\
\hline 1 & $\mathrm{~T}$ & 2 & 0 \\
\hline 2 & $\mathrm{~B}$ & 7 & 0 \\
\hline 3 & $\mathrm{C}$ & 0 & 4 \\
\hline 4 & $\mathrm{~T}$ & 11 & 0 \\
\hline 5 & $\mathrm{~T}$ & 16 & 11 \\
\hline 6 & $\mathrm{~T}$ & 4 & 0 \\
\hline 7 & $\mathrm{~T}$ & 0 & 0 \\
\hline 8 & $\mathrm{C}$ & 0 & 0 \\
\hline 9 & $\mathrm{C}$ & 0 & 0 \\
\hline 10 & $\mathrm{~T}$ & 0 & 1 \\
\hline 11 & $\mathrm{~T}$ & 2 & 4 \\
\hline 12 & $\mathrm{C}$ & 21 & 10 \\
\hline 13 & $\mathrm{~B}$ & 0 & 0 \\
\hline 14 & $\mathrm{~B}$ & 5 & 3 \\
\hline 15 & $\mathrm{C}$ & 0 & 3 \\
\hline 16 & $\mathrm{~T}$ & 15 & 0 \\
\hline 17 & $\mathrm{~B}$ & 0 & 1 \\
\hline 18 & $\mathrm{~B}$ & 0 & 0 \\
\hline 19 & $\mathrm{~T}$ & 6 & 1 \\
\hline 20 & $\mathrm{C}$ & 2 & 0 \\
\hline 21 & $\mathrm{C}$ & 0 & 0 \\
\hline 22 & $\mathrm{C}$ & 7 & 0 \\
\hline 23 & $\mathrm{C}$ & 10 & 0 \\
\hline 24 & $\mathrm{C}$ & 60 & 2 \\
\hline 25 & $\mathrm{C}$ & 3 & 11 \\
\hline 26 & $\mathrm{C}$ & 0 & 0 \\
\hline 27 & $\mathrm{~T}$ & 1 & 1 \\
\hline 28 & $\mathrm{C}$ & 7 & 0 \\
\hline 29 & B & 4 & 14 \\
\hline 30 & B & 23 & 0 \\
\hline 31 & $\mathrm{~T}$ & 2 & 2 \\
\hline 32 & $\mathrm{C}$ & 0 & 2 \\
\hline 33 & $\mathrm{C}$ & 9 & 6 \\
\hline 34 & B & 0 & 0 \\
\hline
\end{tabular}




\section{Testing for Heterogeneous Treatment Effects}

While most experiments are designed to investigate the average effects of a specific treatment of interest on a given outcome, researchers often may wish to investigate differences in the impact of the treatment by sex, ethnicity, income level, or other individual or household characteristics. In the simplest case, one may want to simply investigate one particular interaction of interest (possibly reflecting a particular model prediction or anecdotal evidence from the program rollout); in other instances, the researcher may simply be curious to see which factors modify an intervention's impact. Testing for heterogeneous treatment effects without adjusting the estimated standard errors for multiple testing after the fact, is highly likely to result in incorrect statistical inference. Given that $95 \%$ confidence intervals are constructed to allow for a false discovery probability of 0.05 on each interaction term, the probability of getting k significant pvalues with zero true effects is given by the following binomial distribution:

$$
f(k, m, \alpha)=\left(\begin{array}{l}
m \\
k
\end{array}\right) \alpha^{k}(1-\alpha)^{(m-k)} .
$$

Plugging in for $\alpha=0.05$ and $m=10$, the probabilities of one, two, and more false discoveries with 10 different interaction terms are given by $31.5 \%, 7.5 \%$ and $1.2 \%$ as described in in Table 3 below.

TABLE 3

THEORETICAL (BINOMIAL) Distribution OF FALSE DisCOVERIES WITH 10 RANDOM INTERACTION TERMS AND ALPHA $=0.05$.

\begin{tabular}{lc}
\hline Event & Probability \\
\hline No hypothesis significant & 0.598 \\
One hypothesis significant & 0.315 \\
Two hypothesis significant & 0.075 \\
Three or more hypotheses significant & 0.012 \\
\hline
\end{tabular}


The results shown in Table 3 are valid under the assumption that the interaction terms chosen for empirical analysis are orthogonal to each other. While this may be a reasonable assumption in the case where researchers simply wish to explore interactions with a series of baseline covariates such as sex, urban residence, ethnicity or religious affiliation, the assumption of independent interaction terms appears implausible in cases where researchers pursue a specific model prediction. Hypotheses regarding interaction effects are often generated by structural models developed by the researcher. Since the baseline characteristic of interest (say poverty) may not be easy to measure or directly available in the data, researchers may resort to trying out a series of proxies for the variable of interest, which can be presumed to be correlated. With correlated independent variables tested sequentially, the likelihood of finding one proxy significant will be positively associated with the likelihood of find other proxies used in the regressions significant, so that the basic binomial distribution underlying Table 3 can no longer be used to derive expected distributions of false discoveries.

While generalized false discovery distributions are hard to derive theoretically, it is relatively easy to generate false discovery distributions numerically. In order to illustrate this, we run a series of Monte-Carlo simulations under a range of dependence assumptions. Following the setup in Table 3, we assume that researchers conduct 10 independent tests within each experiment. In our baseline scenario, we assume that variables (interaction terms) are independent. As the first row of Table 4 shows, this yields exactly the distribution of false discoveries predicted by the binomial distribution (Table 3 ), with on average $40 \%$ of regressions showing at least one significant result.

To operationalize correlations among interaction terms, we assume that all included variables contain a common random variable component. One may think of the common random variable as representing the true variable of interest, and of the 10 independent variables as the proxy for this variable; alternatively, one may view the common component as a reflection of the more general dynamics between the variables included.

In order to provide a general sense of the induced changes in the distribution of false discoveries, we simulate a wide range of correlations, starting from a correlation of across proxies of 0.06 
(Table 4, row 2) to a correlation of 0.8 . As Table 4 shows, removing the independence assumption does indeed lower the false discovery risk. The reductions are, however, rather minor under most scenarios. Under the assumption that the 10 proxies display a correlation of 0.25 , the false discovery risk drops from 40 to 36 percent. Even if one is willing to make the assumption that the average correlation is over 0.5 , the false discovery risk remains at $26 \%$, meaning that choosing interactions at random will lead to a more than one in four chance of finding at least one interaction significant at the $95 \%$ level. What is more concerning is that the risk of finding multiple false positives increases substantially with correlated interaction terms. While there is only a 1 in 100 chance of finding 3 or more false positives with 10 randomly chosen interaction terms, the risk of finding multiple false positives exceeds $5 \%$ with correlations over 0.5 . Given that a large number of significant (and close to significant) interaction terms will likely convince even more skeptical researchers and reviewers, the chance of false discoveries being positively reviewed and published may actually be higher than lower with correlated interaction terms.

\section{TABLE 4}

EMPIRICAL Distribution of FALSE Discoveries With CORRELATED INTERACTION TERMS AND ALPHA $=0.05$

\begin{tabular}{ccccc}
$\begin{array}{c}\text { Correlation with } \\
\text { unobservable }\end{array}$ & $\begin{array}{c}\text { Correlation } \\
\text { of proxies }\end{array}$ & $\begin{array}{c}\text { Probability of at least } \\
\text { one false discovery }\end{array}$ & $\begin{array}{c}\text { Probability of two } \\
\text { false discoveries }\end{array}$ & $\begin{array}{c}\text { Probability of three or } \\
\text { more false discoveries }\end{array}$ \\
\hline 0.000 & 0.000 & 40.2 & 8.0 & 1.2 \\
0.250 & 0.063 & 39.1 & 7.3 & 1.5 \\
0.500 & 0.250 & 36.1 & 7.1 & 2.6 \\
0.750 & 0.563 & 26.5 & 5.3 & 5.6 \\
0.900 & 0.810 & 17.1 & 5.3 & 6.5 \\
\hline
\end{tabular}

\section{Heterogeneous Treatment Effects in Practice: PROGRESA}

In order to illustrate how the distribution of estimated heterogeneous treatment effects looks in practice, we randomly test for such effects within the experimental data collected as part of the the Programa de Educación, Salud y Alimentación (PROGRESA). PROGRESA, now called OPORTUNIDADES, is a community-level randomized experiment designed to increase school attendance among the poor through a conditional cash transfer program. By providing a cash 
transfer to poor families large enough to compensate for lost wages from child labor ${ }^{4}$ (Emmanual Skoufias, 2005), the conditional cash transfer program was aiming at changing parental schooling decisions.

While PROGRESA's impact on schooling has been well documented (Paul Schultz, 2004), it seems natural to ask whether the program impact was contingent on, or mediated by, specific household characteristics of interest at baseline. One may, for example, conjecture that the program impact increases with measures of household poverty or vulnerability. The PROGRESA baseline data from the 1997 includes a large array of measures one could use as potential markers for poverty: size of the household, access to piped water and electricity, asset holdings, characteristics of the dwelling, household size and many more. Given the difficulties associated with correctly measuring the income and wealth level of the household, it appears plausible that an interested researcher would consider a larger set of measures, and we shall for simplicity assume that each researcher uses 10 proxies in his analysis. While this may appear high at first sight, 10 interaction terms appear fairly common in the literature: as our review shows, the average paper analyzes 6.4 subgroups and tests for 2.2 interaction effects. The researcher then estimates the following model:

$$
y_{i}=\alpha+\beta T_{i}+\delta W_{i}+\lambda\left(T_{i} x W_{i}\right)+\varepsilon_{i},
$$

where $y_{i}$ is the outcome of interest (in this case schooling), $T$ is the treatment indicator ( 1 if the household was targeted by PROGRESA), $W_{i}$ is one of the 10 poverty indicators coded, and $T_{i} x W_{i}$ is the interaction between the poverty indicator and PROGRESA. While we focus on interaction-term-based empirical models in our simulations, it is easy to see that the results will look virtually the same if separate regressions were conducted for each subgroup of interest.

Given that virtually any baseline variable could be interpreted as a proxy for household poverty or vulnerability, we code all available baseline variables with non-zero variation within the treatment and control groups into binary variables. The total list of binary indicators (148 binary

\footnotetext{
4 A detailed description of the program as well as links to several evaluation studies are available at http://www.ifpri.org/dataset/mexico-evaluation-progresa.
} 
variables) is shown in Appendix Table 2. We assume that each researcher randomly chooses 10 variables out of the set of 148 , and runs 10 separate regressions as described in equation (1). ${ }^{5}$ We run a Monte-Carlo simulation with 10,000 experiments, where we randomly chose a set of 10 out the 148 variables to interact with the treatment in each round. The Monte-Carlo simulation can thus be viewed as an approximation of a setting where a large number of independent researchers work on a given data set, and each of them subjectively chooses 10 variables as proxies for a specific variable of interest. We focus on binary indicators of heterogeneity which is common in the literature. The average correlation across variables is rather low, with a maximum correlation of 0.25 , and an average correlation of 0.03 across the 10 proxies used in each simulation.

The results of the Monte-Carlo experiment are displayed in Figure 1. With 10 random binary regressors from the PROGRESA baseline data, more than $62 \%$ of cases (or independent researchers picking 10 interaction terms) find at least one interaction term significant at the $5 \%$ level; in $17 \%$ of all cases, 2 interaction terms are significant, and in $4 \%$ of cases, 3 or more interaction terms are significant.

\footnotetext{
${ }^{5}$ For data collected to test a specific hypothesis, we might expect that the choices of our hypothetical "researchers" would be correlated instead of being independent. However, evaluating how groups respond differentially to social programs may not always be determined by specific theoretical hypotheses. Even when there are theoretical hypotheses, reasonable people might disagree about the best variables used to proxy for theoretically defined variables such as vulnerability.
} 


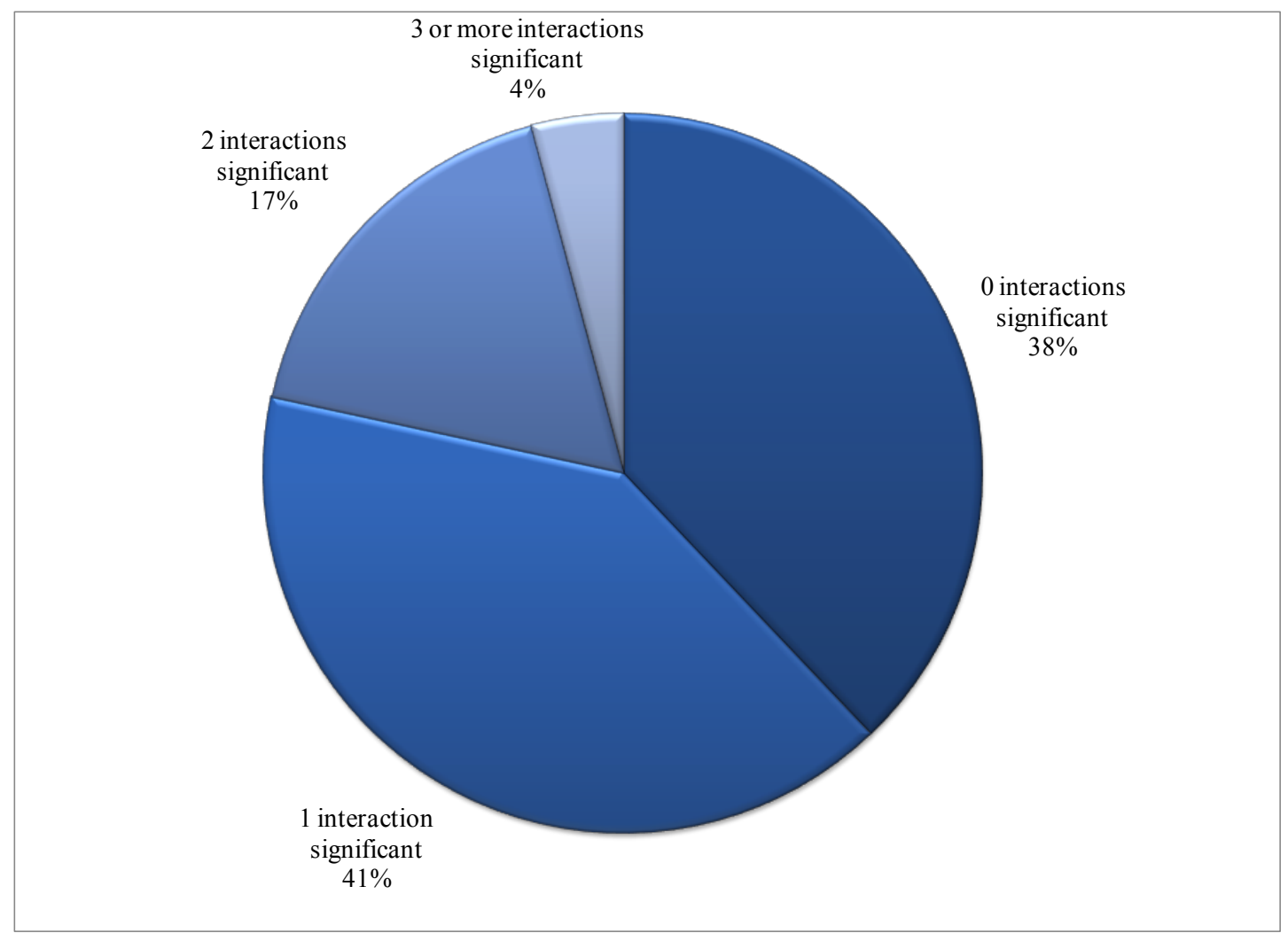

Notes: Based on 10000 random block of size 11, consisting of the main treatment effect and ten randomly selected and independently tested interaction terms.

\section{FIGURE 1}

Empirical Distribution of Significant Coefficients at $\alpha=0.05$

Given that our proxies have relatively low correlation and that our Monte Carlo simulations of theoretical distributions suggest that we would expect at least one significant effect in about $40 \%$ of cases with zero correlation, we expect that over half of the statistically significant coefficients can be presumed to be false discoveries.

\section{Statistical Corrections for Multiple Testing}

Statisticians have been long aware of the problem of multiple hypothesis testing. For the purposes of this paper, we consider the frequentist approach to controlling for multiple testing. 
The probability of the union of two events $A_{1}$ and $A_{2}$ is equal to the sum of the two probabilities $P\left(A_{1}\right)$ and $P\left(A_{2}\right)$ minus the probability of the intersect, i.e.

$$
P\left(A_{1} \cup A_{2}\right)=P\left(A_{1}\right)+P\left(A_{2}\right)-P\left(A_{1} \cap A_{2}\right)
$$

This means that the sum of $P\left(A_{1}\right)$ and $P\left(A_{2}\right)$ constitutes an upper bound of $P\left(A_{1} \cup A_{2}\right)$. If $A_{1}$ and $A_{2}$ describe very similar events, this upper bound may be distant from the true probability; if $A_{1}$ and $A_{2}$ are nearly independent $\left(P\left(A_{1} \cap A_{2}\right) \approx 0\right)$, this upper bound will be very close to the true probability. In the case of multiple events, equation (1) becomes a bit more complex, but the intuition remains exactly the same as in the two events case.

Based on this basic notion, Boole's inequality states that for a finite set of events $A_{1}, \ldots, A_{m}$ the probability of one event happening can never be greater than the sum of the probabilities of each individual event, i.e.

$$
P\left(\bigcup_{i=1}^{m} A_{i}\right) \leq \sum_{i=1}^{m} P\left(A_{i}\right) .
$$

Building on this inequality, the Italian mathematician Carlo Emilio Bonferroni proposed a solution to the multiple testing problem. Assume we want to test $m$ (dependent or independent) hypotheses at level $\alpha$. Boole's inequality ${ }^{6}$ implies that at least one of the hypotheses comes out significant with probability less or equal to $m \alpha$. However, in order to keep the chance of false discoveries (Type I errors) at the desired level, we would like this upper bound to be $\alpha$ and not $m \alpha$. Bonferroni showed that this can be achieved by testing each single hypothesis at the level $\alpha^{\prime}=\alpha / m$. This is called the Bonferroni correction, designed to control the so-called familywise error rate (FWER).

\footnotetext{
${ }^{6}$ Boole's inequality is sometimes also referred to as Bonferroni's inequality.
} 
Duflo et al. (2008a) argue that Bonferroni type corrections may not be very useful in the context of economic field experiments, because the control of Type I errors might come at the cost of high Type II errors (less power). At standard 95\% confidence intervals, testing for 10 effects simultaneously would require marginal p-values of 0.005 for each individual variable. Recent statistical and econometric research has produced a number of alternative methods to correct for multiple hypothesis testing, which are much more powerful than the simple Bonferroni method (Joseph P. Romano and Michael Wolf, 2005). We focus on frequentist methods here but recent work by Gelman, Hill and Yajima (2010) proposes Bayesian multilevel models which can address the problem of multiple comparisons and increase efficiency. Another possibility would be to consider bootstrap approaches to estimating uniform test critical values. Westfall and Young (1994) provide a re-sampling based procedure for correcting for multiple hypothesis testing.

Well known among natural scientists, but still not on the radar of most economists is the multiple testing approach introduced in a seminal paper by Benjamini and Hochberg (Y. Benjamini and Y. Hochberg, 1995a). Rather than focusing on the FWER, the authors define the false discovery rate (FDR) as the expectation of the false discovery proportion (FDP), i.e, the proportion of the rejected null hypothesis that are erroneously rejected. If all null hypotheses are true, the FDR is equivalent to the FWER. Further, if not all null hypotheses are true, it can be shown that any procedure that controls the FWER also controls the FDR. If a procedure controls the FDR only, a gain in power may be expected. The potential for increase is larger when more of the hypotheses are non-true.

Consider testing a set of hypotheses $H_{1}, H_{2}, \ldots, H_{m}$ based on the corresponding pvalues $p_{1}, p_{2}, \ldots, p_{m}$. Let $p_{(1)} \leq p_{(2)} \leq \ldots \leq p_{(m)}$ be the ordered $\mathrm{p}$-values, and denote by $H_{(i)}$ the null hypothesis corresponding to $p_{(i)}$. Let $k$ be the largest $i$ for which

$$
p_{(i)} \leq \frac{i}{m} \alpha
$$


Then reject all $H_{(i)}$ with $i=1,2, \ldots ., k$. Benjamini and Hochberg (1995a) show that this procedure controls the FDR at $\alpha$; independence of the test statistics is not needed for the proof.

It is easiest to illustrate the differences between the FWER and the FDR approaches with an example. Consider an experiment with one treatment, but 10 different dependent variables. The ordered p-values on each of the 10 estimated coefficients look as follows:

$$
\begin{aligned}
& p_{(1)}=0.001, p_{(2)}=0.004, p_{(3)}=0.006, p_{(4)}=0.008, p_{(5)}=0.010 \\
& p_{(6)}=0.040, p_{(7)}=0.050, p_{(8)}=0.060, p_{(9)}=0.100, p_{(10)}=0.400
\end{aligned}
$$

Without any adjustment for multiple testing we reject seven of the ten hypotheses at a 5 percent level of significance. The Bonferroni-adjustment requires a p-value of $\alpha^{\prime}=\frac{0.05}{10}=0.005$, which means that only two out of the ten hypotheses get rejected. With the Benjamini and Hochberg method we check the condition

$$
p_{(i)} \leq \frac{i}{10} \alpha
$$

sequentially starting with $\mathrm{i}=10$. The first $\mathrm{p}$-value to satisfy the condition is $p_{(5)}$ with $0.01<$ 0.025; it is straightforward to see that the condition is also satisfied for any $i<5$, so that the FDR adjustment leads to a rejection of 5 out of the 10 tested hypotheses.

Genovese and Wasserman (2006) show that the Benjamini and Hochberg method is optimal in the sense that it minimizes the false non-discovery rate (FNR) subject to the constraint that the FDR is controlled at level $\alpha$, where the FNR is defined as the expectation of the proportion of non-rejections that are incorrect. In other words, the Benjamini and Hochberg method keeps the number of type II errors as small as possible, i.e. the chance of not rejecting a hypothesis when it is false. While this correction is generally less conservative than the methods based on FWER 
(which continue to be most frequently used), it is also subject to the criticism of the independent test assumptions. While correction models under more general or arbitrary dependence structures would clearly be desirable, such approaches have not yet been developed (Joseph P. Romano and Michael Wolf, 2005).

\section{Correcting for Multiple Hypotheses Tests in Practice}

There are two important aspects to consider when it comes to the applicability of these correction procedures: 1) the technical knowledge required for implementation; 2) the statistical and empirical consequences in terms of type I and type II errors. The first aspect is fortunately straightforward. Thanks to the multproc package available as a user-written add-on in Stata ${ }^{\mathcal{O}}$ (Roger Newson, 2003), both the FDR and FWER methods are easily implemented in practice. The multproc package takes p-values from a set of variables (from single or multiple regressions) as inputs, and calculates corrected critical p-values for a range of correction procedures. The FWER correction is intuitive, as critical p-values are simply divided by the number of hypotheses tested $(\mathrm{m})$. In the case of single control variable and one interaction term, this implies that the critical $p$-value for significance at the $95 \%$ level shifts from 0.05 to $0.05 / 2=0.025$. It is easy to see that this adjustment keeps the likelihood of a false discovery at the desired low level independent of the number of hypotheses tested.

The FDR adjustment is slightly more complex since it is taking true discoveries into account, and, as a result, adjusts the p-values to a lesser extent than the FWER method. To see how well these adjustments work, we show the implications of the adjustment with truly independent variables (theoretical binomial) in a first step, and then revisit the PROGRESA results presented in Section 3.

Given that the FDR deviates from the FWER correction only if at least one hypothesis is false, it is easy to see that the two corrections have virtually the same effect if we assume 10 independent 
interactions without true effect. With $\alpha=0.05$ and $m=10$ the adjusted p-value under both correction models can be approximated by ${ }^{7}$

$$
p_{a d j}=\frac{\alpha}{m}=\frac{0.05}{10}=0.005
$$

With the adjusted probability of 0.005 , we can get the joint distribution within blocks of 10 by plugging the adjusted p-value into the corresponding binomial distribution. As the results displayed in Table 4 show, the chance of false discoveries is indeed reduced to just below 5\%.

TABLE 4

Theoretical (BINOMIAL) Distribution of FALSE Discoveries WITH 10 RANDOM INTERACTION TERMS AND ALPHA $=0.005$

$\begin{array}{ll}\text { Event } & \text { Probability }\end{array}$

No hypothesis significant

0.951

One hypothesis significant

0.048

Two or more hypothesis significant

0.010

The interpretation of the FDR differs from the typical statistical testing done by economists. The FDR correction is probabilistic, meaning that with a critical value of 0.05 , we expect $5 \%$ of our tested hypotheses to be significant by chance. However, since the number of hypotheses generated by most experiments is relatively small, we will end up concluding that we expect some fraction of a hypothesis to be rejected erroneously, which can be difficult to interpret conceptually. Nonetheless, we would argue that the FDR correction allows for an appropriate level of caution in interpreting the results of testing many hypotheses, without becoming so conservative that we can no longer draw important conclusions about heterogeneity in responses to treatment.

\footnotetext{
${ }^{7}$ Technically, the FDR calculates separate p-values for each hypothesis. The adjusted FWER p-value corresponds to the $\mathrm{p}$-value for the variable with the lowest $\mathrm{p}$-value. The $\mathrm{p}$-value of the second variable would be is 0.01 , the third 0.015 and so on. In practice the first one will already rule out $95 \%$ such that the result is very similar to FWER, but it is not the same.
} 
To provide a better sense of how powerful these corrections are in practice, we show the PROGRESA results displayed in Figure 1 with corrected p-values in Figure 2 below. The assumption underlying the correction is that within each experiment we test 11 hypotheses, the main treatment variable plus 10 randomly selected interaction terms. As Figure 2 shows, neither correction affects the significance of the main treatment effect, which is significant in all cases with both corrections. Large differences emerge, however, with respect to the interaction terms. While we see at least one significant result in $62 \%$ of the specifications if no correction is applied, the likelihood of finding a statistically significant results drops by $60 \%$ (FDR) and $70 \%$ (FWER), respectively, after the correction is applied. This, however, does not mean that researchers applying either correction will never find significant results - as Figure 2 clearly illustrates, the chance of finding one or more significant results in a Table showing 10 interaction terms in the PROGRESA sample is $18 \%$ with the Bonferroni FWER correction, and 24\% with the Benjamini-Hochberg FDR correction.

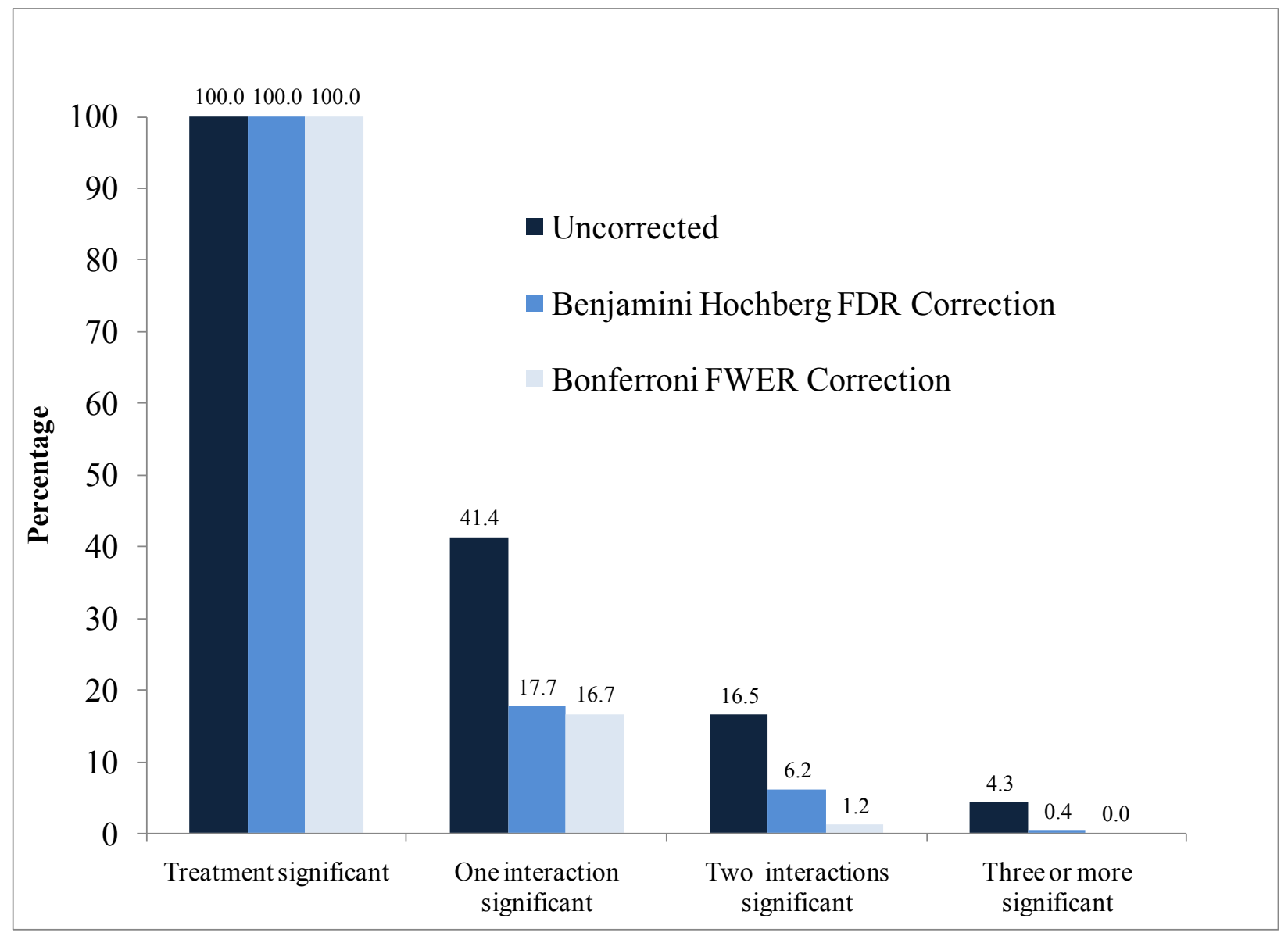

FIGURE 2 


\section{Empirical Distribution of Significant Results with and without Corrections}

\section{ImPlications for Study Design AND POWER CalCulations}

Given that interaction terms may often be of major importance of researchers designing an experiment, one of the key questions is how much of an adjustment is needed to sample size exante if the researcher plans to test for interaction effects ex-post. To understand what the two corrections imply in terms of power, we show a set of numerical simulations in this section. As a first step, we assume a sample size of 2000, with a corresponding (unadjusted) power of 0.5 and investigate how much power is lost in expected terms once we adjust for multiple hypotheses testing. As Figure 3 illustrates, the power drops in a non-linear fashion from 0.35 to about 0.2 for the FDR approach; as expected the drop is larger for the FWER approach, where the power drops to 0.1 if 10 hypotheses are tested simultaneously.

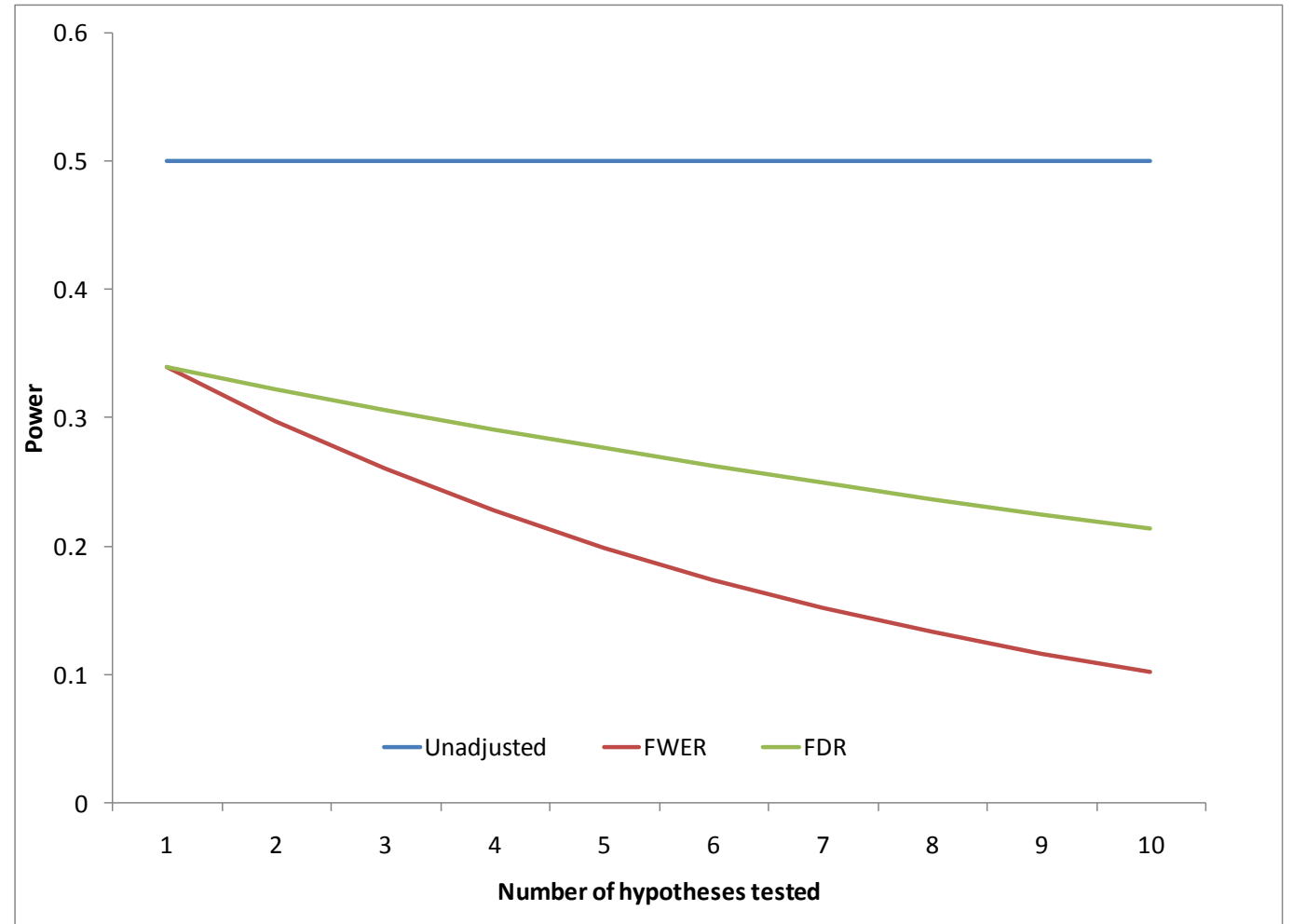

FIGURE 3

Power with Corrections 
While these losses in terms of power may dissuade researchers from applying these corrections in practice, the necessary sample adjustment may not be as large as one may think (or fear) as long as the number of interactions the researcher is interested in is reasonably small.

Figures 4 illustrate this point for one and two control variables and their interaction terms, respectively. As pointed out before, standard power calculations do not apply here as two separate treatment groups lower effective group sizes, and increase estimated standard errors. As Figure 6 shows, the power of the study with sample size 5000 and a treatment effect of 0.05 is 0.9 if no interaction term is included. With the interaction term, the power drops to about 0.7. The drop in power due to the multiple testing corrections is comparable in magnitude. With a sample of 5000, the power with one interaction term drops to about 0.5 , while the power with two interaction terms is about 0.45 .

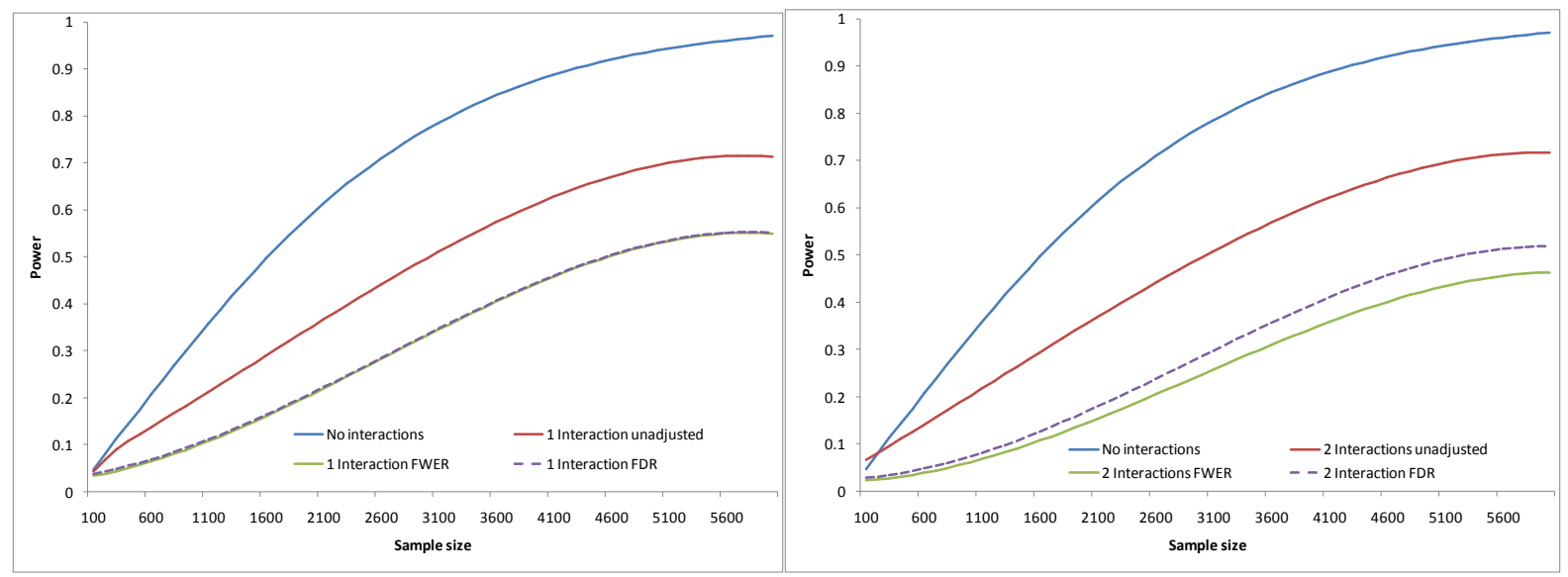

FIGURE 4

Power with one and two Interaction Terms

To see the implications for study design, we show necessary samples with and without corrections for multiple testing in Figure 5 below. As the figure shows, the absolute differences in sample size are rather small for large effect sizes; in relative terms, doing the FWER adjustment implies an average increase in necessary sample size (assumed power is 0.9 ) of about 28 percent with one interaction, 55\% with two interactions, and about $67 \%$ with three 
interactions. ${ }^{8}$ These adjustments are not trivial and may appear overly conservative. As discussed earlier, the FWER adjustment reduces the risk of false discoveries under the most conservative assumption of independence across events. Smaller sample size adjustments could in theory be generated by using the FDR approach and by relaxing the assumption regarding event independence; however, this would require imposing a large set of additional distributional assumptions researchers will struggle to make during early stages of field experiments. From a pragmatic perspective, it seems best to base initial sample size calculations on FWER adjusted standard errors. The FWER adjustment will keep the risk of false discoveries at the desired low levels and guarantee sufficient power for either FWER or FDR standard error adjustments expost.

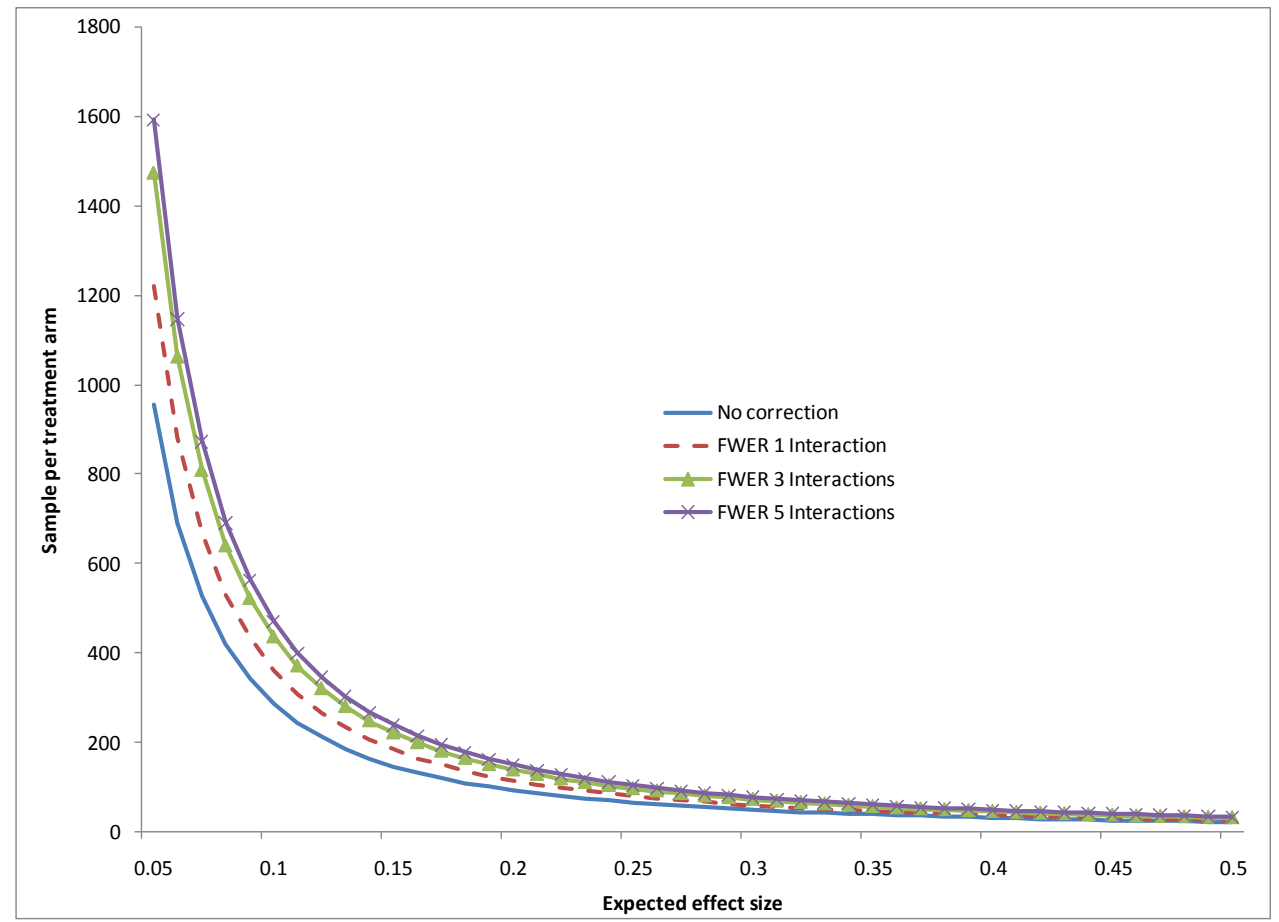

FIGURE 5

Absolute Sample Size with and without FWER Correction

\footnotetext{
${ }^{8}$ We assume that each interaction term is tested separately, so that each regression yields 3 coefficients (covariate, treatment and covariate*treatment) and $3 \mathrm{p}$-values that need to be adjusted.
} 


\section{DisCuSSION AND CONCLUSIONS}

Even though the testing of heterogeneous treatment effects is very common in current empirical work based on experimental data, multiple testing corrections are generally not applied. In this paper we demonstrate that standard statistical inference is not valid when multiple heterogeneous treatment effects are tested, and that ignoring this issue is likely to generate a large number of false discoveries. Without any true effect, the likelihood of finding at least one result significant at the 5\% level is $40 \%$ with 10 uncorrelated interaction effects. In the PROGRESA example, we find that more than half of the results significant at the $5 \%$ level can be assumed to reflect false discoveries. This risk appears high and, more importantly, unnecessary given the readily available correction models developed in the statistics literature.

There are three main critiques of the correction procedures discussed here. The first concern regards the actual reporting of statistical tests conducted. Even if proper adjustments are applied to the final set of interaction terms reported, the underlying variable selection is unobservable expost, and may itself be the result of pre-testing. One possible approach to address this issue might be a central registration system similar to the ones used in medical trials. In fact, Duflo et al (2008a) suggest that granting agencies create such a database of projects and their ex-ante designs. ${ }^{9}$

The second concern relates to the definition of what counts as a distinct hypothesis. Given that many variables may be used as proxies for a specific factor of interest such as income or human capital, it may be tempting to argue that all interacted variables are related, and thus reflect one single hypothesis. However, given that the correlation between any two proxies of interest is small in most cases empirically, ${ }^{10}$ treating multiple measures of a specific factor of interest as single hypothesis appears not advisable from a statistical perspective. Furthermore, our analysis shows that correlation of interaction terms does not solve the problem of false discoveries and may in fact make it worse in some cases.

\footnotetext{
${ }^{9}$ Some researchers have begun to publish their analysis prior to conducting experiments. Another potential alternative is the use of interdisciplinary system where researchers in any field can currently post their designs prior to conducting experiments, such as http://clinicaltrials.gov/.

${ }^{10}$ In the PROGRESA data, the highest correlation between any two indicator variables is 0.27.
} 
Finally and maybe most importantly, there is the concern that applying more stringent standard errors increases the chance of type-II errors, i.e. the chance of not rejecting a hypothesis when it is false. While adjusting p-values clearly comes with some loss of power, we have shown in this paper that the cost in terms of additional sample size required for researchers planning to test for heterogeneous treatment effects ex-post appears well worth the benefit in terms of reduced false discovery risk.

Overall, a wider application of multiple testing procedures in the economics literature appears highly desirable. Testing for heterogeneous treatment effects is of obvious interest to researchers, and neither can, nor should, be avoided in practice. The resulting risk of false discoveries is high, but can be reduced to a minimum if correction procedures are applied. 


\section{Appendix Table 1: Index of Strict Natural Experiments}

A Field Experiment in Charitable Contribution: The Impact of Social Information on the Voluntary Provision of Public 1 Goods

2 Credit Elasticities in Less-Developed Economies: Implications for Microfinance

3 Do Workers Work More if Wages Are High? Evidence from a Randomized Field Experiment

$4 \quad$ Does Job Corps Work? Impact Findings from the National Job Corps Study

5 Does Price Matter in Charitable Giving? Evidence from a Large-Scale Natural Field Experiment

6 Experimental Analysis of Neighborhood Effects

7 Gift-Exchange in the Field

8 How High are Rates of Return to Fertilizer? Evidence from Field Experiments in Kenya

9 Incentives for Managers and Inequality Among Workers: Evidence From a Firm-Level Experiment

10 Incentives to Exercise

11 Incentives to Learn

12 Information, School Choice, and Academic Achievement: Evidence From Two Experiments

13 Insurance, credit, and technology adoption: Field experimental evidence from Malawi

14 Intra-household allocation of free and purchased mosquito nets

15 Monitoring Corruption: Evidence from a Field Experiment in Indonesia

16 Neighborhood Effects on Crime for Female and Male Youth: Evidence From a Randomized Housing Voucher Experiment

17 Observational Learning: Evidence from a Randomized Natural Field Experiment

18 Obtaining a Driver's License in India: an Experimental Approach to Studying Corruption

19 Power to the People: Evidence From a Randomized Field Experiment on Community-Based Monitoring in Uganda

20 Powerful Women: Does Exposure Reduce Bias

21 Putting Behavioral Economics to Work: Testing for Gift Exchange in Labor Markets Using Field Experiments

22 Remedying Education: Evidence From Two Randomized Experiments in India

23 Requiring a Math Skills Unit: Results of a Randomized Experiment

24 Resource and Peer Impacts on Girls’ Academic Achievement: Evidence from a Randomized Experiment

25 Returns to Capital in Microenterprises: Evidence From a Field Experiment

26 Salience and Taxation: Theory and Evidence

27 Saving Incentives for Low- and Middle-Income Families: Evidence From a Field Experiment with H \& R Block

28 Social Connections and Incentives in the Workplace: Evidence from Personnel Data

29 The Demand for, and Impact of, Learning HIV Status

30 The Effects of High Stakes High School Achievement Awards: Evidence from a Randomized Trial

31 The importance of being informed: Experimental evidence on demand for environmental quality

32 Toward an Understanding of the Economics of Charity: Evidence From a Field Experiment

33 Tying Odysseus to the Mast: Evidence From a Commitment Savings Product in the Philippines

34 What Matters (and What Does Not) in Households' Decision-Making Regarding Investments in Malaria Prevention? 


\section{Appendix Table 2: Binary Variables Used in PROGRESA Regressions}

\begin{tabular}{|c|c|c|c|}
\hline 1 bathroom & 38 head_nospanish & 75 lost_limb & 112 rooftype 4 \\
\hline 2 bathroom_water & 39 head_o60 & 76 mental & 113 rooftype 5 \\
\hline 3 bedrooms 1 & 40 head_perm_unable & 77 migrant & 114 rooftype 6 \\
\hline 4 bedrooms 2 & 41 head_preparatoria & 78 needs_help_to_move & 115 rooftype 7 \\
\hline 5 bedrooms 3 & 42 head_primary & 79 no_waterelectric & 116 rooftype 8 \\
\hline 6 bedrooms 4 & 43 head_primary_income & 80 noincome & 117 rooftype 9 \\
\hline 7 bedrooms 5 & 44 head_profesional & 81 not_childofhead & 118 second_income \\
\hline 8 bedrooms6plus & 45 head_retired & 82 one_child & 119 shared_building \\
\hline 9 blender & 46head_secondary & 83 owns_agri_land & 120 spouse_away \\
\hline 10 blind & 47 head single & 84 owns animals & 121 stove \\
\hline 11 budget_control_head & 48 head_single_female & 85 owns_cattle & 122 three_children \\
\hline 12 budget_control_other & 49 head_socialsecurity & 86 owns_chicken & 123 treatment_dif \\
\hline 13 budget_control_shared & 50 head_temp_unable & 87 owns_donkey & 124 treatment_hospital \\
\hline 14 budget_control_spouse & 51 head_u20 & 88 owns_goatsorsheeps & 125 treatment_imss \\
\hline 15 car & 52 head_widowed & 89 owns_horse & 126 treatment_issste \\
\hline $16 \mathrm{~cd}$ & 53 head_working & 90 owns_land & 127 treatment_othergov \\
\hline 17 deaf & 54hhsize10plus & 91 owns_multiple_pieces & 128 treatment_ssa \\
\hline 18decision_head & 55 hhsize2 & 92 owns_ox & 129 truck \\
\hline 19 dumb & 56 hhsize 3 & 93 owns_pigs & $130 \mathrm{tv}$ \\
\hline 20 electric_lights & 57 hhsize 4 & 94 owns_rabbits & 131 two_children \\
\hline $21 \mathrm{fan}$ & 58 hhsize 5 & 95 piped_inside & 132 video \\
\hline 22 father_athome & 59 hhsize6 & 96 piped_water & 133 walltype 1 \\
\hline 23 female & 60 hhsize 7 & 97 radio & 134 walltype 10 \\
\hline 24 five_or_more_children & 61 hhsize8 & 98 receives_apoyoINI & 135 walltype 11 \\
\hline 25 floortype 1 & 62 hhsize 9 & 99 receives_becacapicaticaion & 136 walltype 12 \\
\hline 26 floortype 2 & 63 house_paid & 100 receives_desayuno_escolar & 137 walltype 13 \\
\hline 27 floortype 3 & 64 house_paying & 101 receives_despensa_DIF & 138 walltype 14 \\
\hline 28 floortype 4 & 65 house_provided & 102 receives_empleotemporal & 139 walltype 15 \\
\hline 29 four_children & 66 house_rented & 103 receives_leche & 140 walltype 2 \\
\hline 30 fridge & 67 inshool_97 & 104 receives_ninosdesolid & 141 walltype 3 \\
\hline 31 head_2060 & 68 kids_medical_head & 105 receives_tortilla & 142 walltype 4 \\
\hline 32 head_basica & 69 kids_medical_other & 106 rooftype 1 & 143 walltype 5 \\
\hline 33 head_dialect & 70kids_medical_shared & 107 rooftype 10 & 144 walltype 6 \\
\hline 34head_female & 71 kids_medical_spouse & 108 rooftype 11 & 145 walltype 7 \\
\hline 35 head_literate & 72 Laundry & 109 rooftype 12 & 146 walltype 8 \\
\hline 36head_married & 73 light_meter & 110 rooftype 2 & 147 walltype 9 \\
\hline 37 head noschool & 74 Literate & 111 rooftype 3 & 148 water heater \\
\hline
\end{tabular}




\section{REFERENCES}

Angrist, Joshua and Victor Lavy. 2009. "The Effects of High Stakes High School Achievement Awards: Evidence from a Randomized Trial." American Economic Review, 99((4), 1384-414.

Angrist, Joshua D. 2004. "Treatment Effect Heterogeneity in Theory and Practice " The Economic Journal, 114 (March), C52-C83.

Ariely, Dan; Uri Gneezy; George Loewenstein and Nina Mazar. 2009. "Large Stakes and Big Mistakes." Review of Economic Studies, 76(2), 451-69.

Ashraf, Nava. 2009. "Spousal Control and Intra-Household Decision Making: An Experimental Study in the Philippines." American Economic Review, , (), pp. . 99(4), 1245-77.

Ashraf, Nava; Dean Karlan and Wesley Yin. 2006. "Tying Odysseus to the Mast: Evidence from a Commitment Savings Product in the Philippines." The Quarterly Journal of Economics, 121(2), 635-72.

Bandiera, Oriana; Iwan Barankay and Imran Rasul. 2007. "Incentives for Managers and Inequality among Workers: Evidence from a Firm-Level Experiment." The Quarterly Journal of Economics, 122(2), 729-73.

Bandiera, Oriana; Iwan Barankay and Imran Rasul. 2009. "Social Connections and Incentives in the Workplace: Evidence from Personnel Data." Econometrica, 77(4), 1047-94.

Banerjee, Abhijit V.; Shawn Cole; Esther Duflo and Leigh Linden. 2007. "Remedying Education: Evidence from Two Randomized Experiments in India." The Quarterly Journal of Economics, 122(3), 1235-64.

Beaman, Lori; Raghabendra Chattopadhyay; Esther Duflo; Rohini Pande and Petia Topalova. 2009. "Powerful Women: Does Exposure Reduce Bias?" The Quarterly Journal of Economics, 124(4), 1497-540.

Benjamini, Y. and Y. Hochberg. 1995a. "Controlling the False Discovery Rate: A Practical and Powerful Approach to Multiple Testing." Journal of the Royal Statistical Society Series B, 57(1), 289--300.

Benjamini, Y. and Y. Hochberg. 1995b. "Controlling the False Discovery Rate: A Practical and Powerful Approach to Multiple Testing." Journal of the Royal Statistical Society Series, B(57), 289--300.

Bertrand, Marianne; Simeon Djankov; Rema Hanna and Sendhil Mullainathan. 2007. "Obtaining a Driver's License in India: An Experimental Approach to Studying Corruption." The Quarterly Journal of Economics, 122(4), 1639-76.

Bitler, Marianne P.; Jonah B. Gelbach and Hilary W. Hoynes. 2006. "What Mean Impacts Miss: Distributional Effects of Welfare Reform Experiments." American Economic Review, 96(4), 988-1012.

Björkman, Martina and Jakob Svensson. 2009. "Power to the People: Evidence from a Randomized Field Experiment on Community-Based Monitoring in Uganda." The Quarterly Journal of Economics, 124(2), 735-69.

Blumenschein, Karen; Glenn C. Blomquist; Magnus Johannesson; Nancy Horn and Patricia Freeman. 2008. "Eliciting Willingness to Pay without Bias: Evidence from a Field Experiment." Economic Journal, 118(525), 114-37.

Boisjoly, Johanne; J. Duncan Greg; Michael Kremer; Dan M. Levy and Jacque Eccles. 2006. "Empathy or Antipathy? The Impact of Diversity." American Economic Review, 96(5), 1890-905.

Carpenter, Jeffrey; Jessica Holmes and PeterHans Matthews. 2008. "Charity Auctions: A Field Experiment." Economic Journal, 118(525), 92-113.

Charness, Gary and Uri Gneezy. 2009. "Incentives to Exercise." Econometrica, 77(3), 909-31.

Duflo, Esther; William Gale; Jeffrey Liebman; Peter Orszag and Emmanuel Saez. 2006. "Saving Incentives for Low- and Middle-Income Families: Evidence from a Field Experiment with H\&R Block." The Quarterly Journal of Economics, 121(4), 1311-46.

Duflo, Esther; Rachel Glennerster and Michael Kremer. 2008a. "Using Randomization in Development Economics Research: A Toolkit," Handbook of Development Economics. 3895--962. 
Duflo, Esther; Michael Kremer and Jonathan Robinson. 2008b. "How High Are Rates of Return to Fertilizer? Evidence from Field Experiments in Kenya." American Economic Review Papers and Proceedings, 98(2), 482-88.

Dupas, Pascaline. 2009. "What Matters (and What Does Not) in Households' Decision to Invest in Malaria Prevention?" American Economic Review Papers and Proceedings, 99(2), 224-30.

Engemann, Kristie M. and Howard J. Wall. 2009. "A Journal Ranking for the Ambitious Economist." Federal Reserve Bank of St. Louis Review., 91(3), 127-39.

Falk, Armin. 2007. "Gift Exchange in the Field." Econometrica, 75(5), 1501-11.

Farcomeni, A. . 2008. "A Review of Modern Multiple Hypothesis Testing, with Particular Attention to the False Discovery Proportion. ." Statistical Methods in Medical Research, 17, 47--388.

Fearon, James D.; Macartan Humphreys and Jeremy M. Weinstein. 2009. "Can Development Aid Contribute to Social Cohesion after Civil War? Evidence from a Field Experiment in Post-Conflict Liberia." American Economic Review Papers and Proceedings, 99(2), 287-91.

Fehr, Ernst and Lorenz Goette. 2007. "Do Workers Work More If Wages Are High? Evidence from a Randomized Field Experiment." American Economic Review, 97(1), 298-317.

Fisman, Raymond; Sheena S. Iyengar; Emir Kamenica and Itamar Simonson. 2006. "Gender Differences in Mate Selection: Evidence from a Speed Dating Experiment." The Quarterly Journal of Economics, 121(2), 673-97.

Gelman, A.; J. Hill and M.Yajima. 2010. "Why We (Usually) Don't Worry About Multiple Comparisons." Mimeo.

Genovese, C. and L. Wasserman. 2006. "Exceedance Control of the False Discovery Proportion." Journal of the American Statistical Association, 101, 1408--17.

Genovese, C. and L. Wasserman. 2004. "A Stochastic Process Approach to False Discovery Control." Annals of Statistics, (32), 1035--61.

Gin, Xavier and Dean Yang. 2009. "Insurance, Credit, and Technology Adoption: Field Experimental Evidencefrom Malawi." Journal of Development Economics, 89(1), 1-11.

Gneezy, Uri and John A. List. 2006. "Putting Behavioral Economics to Work: Testing for Gift Exchange in Labor Markets Using Field Experiments." Econometrica, (), pp. . 74(5), 1365-84.

Green, Donald P. and Holger L. Kern. 2010. "Modeling Heterogeneous Treatment Effects in Large-Scale Experiments Using Bayesian Additive Regression Tree." Mimeo.

Hahn, Jinyong; Keisuke Hirano and Dean Karlan. 2011. "Adaptive Experimental Design Using the Propensity Score." Journal of Business \& Economic Statistics, 29(1), 96-108.

Harrison, Glenn W. and John A. List. 2004. "Field Experiments." Journal of Economic Literature, 42(4), 1009-55.

Hastings, Justine S. and M. Weinstein Jeffrey. 2008. "Information, School Choice, and Academic Achievement: Evidence from Two Experiments." The Quarterly Journal of Economics, 123(4), 1373-414.

Hoffmann, Vivian. 2009. "Intrahousehold Allocation of Free and Purchased Mosquito Nets." American Economic Review Papers and Proceedings, 99(2), 236-41.

Imai, Kosuke and Aaron Strauss. 2011. "Estimation of Heterogeneous Treatment Effects from Randomized Experiments, with Application to the Optimal Planning of the Get-out-the-Vote Campaign." Political Analysis, 19(1), 1-19.

JPAL. 2011. "Abdul Latif Jameel Poverty Action Lab," Cambridge, MA:

Jyotsna, Jalan and E. Somanathan. 2008. "The Importance of Being Informed: Experimental Evidence on Demand for Environmental Quality." Journal of Development Economics, 87(1), 14-28.

Karlan, Dean and John A. List. 2007. "Does Price Matter in Charitable Giving? Evidence from a LargeScale Natural Field Experiment." American Economic Review, 97(5), 1774-93.

Kling, Jeffrey R.; Jeffrey B. Liebman and Lawrence F. Katz. 2007. "Experimental Analysis of Neighborhood Effects." Econometrica, 75(1), 83-119. 
Kling, Jeffrey R.; Jens Ludwig and Lawrence F. Katz. 2005. "Neighborhood Effects on Crime for Female and Male Youth: Evidence from a Randomized Housing Voucher Experiment." The Quarterly Journal of Economics, 120(1), 87-130.

Kremer, Michael and Edward Miguel. 2007. "The Illusion of Sustainability." The Quarterly Journal of Economics, 122(3), 1007-65.

Kremer, Michael; Edward Miguel and Rebecca Thornton. 2009. "Incentives to Learn." The Review of Economics and Statistics, 91(3), 437-56.

Landry, Craig E.; Andreas Lange; John A. List; Michael K. Price and Nicholas G. Rupp. 2006. "Toward an Understanding of the Economics of Charity: Evidence from a Field Experiment." The Quarterly Journal of Economics, 121(2), 747-82.

Lee, David S. 2009. "Training, Wages, and Sample Selection: Estimating Sharp Bounds on Treatment Effects." Review of Economic Studies, 76(3), 1071-102.

List, John A. 2011. "Field Experiments,"

Mel, Suresh de; David McKenzie and Christopher Woodruff. 2008. "Returns to Capital in Microenterprises: Evidence from a Field Experiment." The Quarterly Journal of Economics, 123(4), 132972.

Newson, Roger. 2003. "Multiple-Test Procedures and Smile Plots." The Stata Journal, 3(2), 100-32.

Olken, Benjamin A. 2007. "Monitoring Corruption: Evidence from a Field Experiment in Indonesia." Journal of Political Economy, 115, 200-49.

Pozo, Susan and Charles A. Stull. 2006. "Requiring a Math Skills Unit: Results of a Randomized Experiment." American Economic Review Papers and Proceedings, 96(2), 437-41.

Romano, Joseph P.; Azeem M. Shaikh and Michael Wolf. 2010a. "Hypothesis Testing in Econometrics." Annual Reviews of Economics, 2, 75 -104.

Romano, Joseph P.; Azeem M. Shaikh and Michael Wolf. 2010b. "Multiple Testing"," New Palgrave Dictionary of Economics.

Romano, Joseph P. and Michael Wolf. 2005. "Stepwise Multiple Testing as Formalized Data Snooping." Econometrica, 73(4), 1237-82.

Schochet, Peter Z.; John Burghardt and Sheena McConnell. 2008. "Does Job Corps Work? Impact Findings from the National Job Corps Study." American Economic Review, 98(5), 1864-86.

Schultz, Paul. 2004. "School Subsidies for the Poor: Evaluating the Mexican Progresa Poverty Program." Journal of Development Economics, 74(1), 199-250.

Shang, Jen and Rachel Croson. 2009. "A Field Experiment in Charitable Contribution: The Impact of Social Information on the Voluntary Provision of Public Goods." Economic Journal, 119(540), 1422-39.

Skoufias, Emmanual. 2005. Progresa and Its Impacts on the Welfare of Rural Households in Mexico. Washington, D.C.: IFPRI.

Thornton, Rebecca L. 2008. "The Demand for, and Impact of, Learning Hiv Status." American Economic Review, 98(5), 1829-63.

Todd, Petra E. and Kenneth I. Wolpin. 2006. "Assessing the Impact of a School Subsidy Program in Mexico: Using a Social Experiment to Validate a Dynamic Behavioral Model of Child Schooling and Fertility." American Economic Review, 96(5), 1384-417.

van der Laan, M.; S. Dudoit and K. Pollard. 2004. "Augmentation Procedures for Control of the Generalized Family-Wise Error Rate and Tail Probabilities for the Proportion of False Positives." Statistical Applications in Genetics and Molecular Biology, 3(1), 15.

Whitmore, Diane. 2005. "Resource and Peer Impacts on Girls' Academic Achievement: Evidence from a Randomized Experiment." American Economic Review Papers and Proceedings, 95(2), 199-203. 